\title{
Comparación de herramientas de evaluación de la competencia comunicación oral efectiva
}

\author{
O. Sahuquillo ${ }^{\text {, A. A. Sonseca }}{ }^{\text {b }}$ J. Martínez ${ }^{c}$ y J. Carballeira ${ }^{d}$ \\ a (ossana@upvnet.upv.es), (agsonol@posgrado.upv.es), \\ c(jomarc12@mcm.upv.es), ${ }^{d}$ (jacarmo@mcm.upv.es) \\ Departamento de Ingeniería Mecánica y de Materiales \\ Universitat Politècnica de València, Camino de Vera s/n, 46022, Valencia
}

\begin{abstract}
With the main objective of evaluating oral communication skills, a coevaluation methodology has been carried out for the last years to obtain quantitative results from data collected in the lab sessions on Materials Science, from the Bachelor degree in Chemical Engineering. The evaluation methodology followed is focus on students encouragement to participate more actively in the continuous learning of the subject. Students were asked to prepare oral presentations to explain to the class a summary about a previous lesson. These presentations were evaluated by a rubric-based method. The evaluation was done by using two different rubrics. The first one developed by the authors as a part of a Universitat Politècnica de Valencia project in educational innovation and improvement (PIME); and the second one an institutional one. The last one was developed later at UPV and also based on the experience derived from PIMEs projects. The idea is to compare both of them to prove if there is a good correlation between them.
\end{abstract}

Keywords: generic competences, oral communication, rubric

\footnotetext{
Resumen

Con el objetivo principal de evaluar la competencia de comunicación oral, se ha empleado durante los últimos años una metodología basada en la coevaluación, para obtener resultados cuantitativos de las sesiones de laboratorio de la asignatura Ciencia de Materiales del Grado en Ingeniería Química. La metodología pretende que los alumnos participen más activamente la evaluación continua de la asignatura. Los alumnos tienen que preparar y realizar una presentación oral resumen de la sesión anterior al resto de sus compañeros. La presentación será evaluada usando una rúbrica como herramienta de evaluación. La evaluación fue realizada utilizando dos rúbricas diferentes, una de ellas diseñada por los autores como parte del "Proyecto de Innovación y Mejora Educativa" (PIME) de la Universitat Politècnica de València (UPV). Otra desarrollada posteriormente en la
} 
UPV, basándose en la experiencia derivada de diferentes PIMEs. Se busca comparar si hay una buena correlación entre los resultados obtenidos con ambas rúbricas.

Palabras clave: competencias transversales, comunicación oral, rúbrica

\section{Introducción}

Actualmente en las titulaciones universitarias se ha evolucionado hacia un modelo en el que parece que no hay duda en considerar las competencias transversales como parte fundamental del curriculum de los estudiantes universitarios, dirigiéndose de este modo hacia una formación más completa. Los programas de Grado y Máster ofertados por la Universitat Politècnica de València (UPV), dentro del marco de la Espacio Europeo de Educación Superior, siguen un enfoque basado en competencias [Sursock (2010), Rieckmann (2012)].

Dentro de las competencias se puede diferenciar entre competencias de tipo específico y transversales. Las de tipo específico son las que se han venido evaluando hasta ahora y en las que se tiene gran experiencia en cómo realizarlo. En los últimos años ha surgido la necesidad de evaluar las competencias transversales y donde profesores y las instituciones están enfocando su esfuerzo y recursos.

El presente estudio es un continuación en el trabajo iniciado dentro del marco del "Proyecto de Innovación y Mejora Educativa (PIME Project B12/14: Desarrollo de estrategias para la evaluación de competencias transversales en asignaturas de Ingeniería Mecánica y de Materiales" desarrollado en la UPV. Cómo parte de la primera etapa de este proyecto, se asumió la necesidad de buscar y desarrollar herramientas para la evaluación de las habilidades en comunicación oral por parte de los alumnos [Carballeira (2015)].

En ese momento no se disponía de herramientas de evaluación institucionales para ese propósito. De ese modo fue una oportunidad para el desarrollo de experiencias en este área, junto con soporte a nivel institucional en la UPV mediante programas para la motivación y apoyo a las investigaciones y experiencias relacionadas con este tema [UPV (2014)]. Algunos de los resultados de este proyecto PIME fueron el desarrollo de una metodología y de herramientas para la evaluación de la habilidad en la comunicación basado en un método de rúbricas, teniendo en consideración que la comunicación está considerada como un punto clave de acuerdo al proyecto Tuning (2014).

\section{Objetivos}

El objetivo primordial se centra en evaluar competencias transversales, y más concretamente las habilidades en comunicación oral por parte de los alumnos.

Desde esa premisa, se plantea por un lado el ser capaces de desarrollar herramientas que permitan llevar a cabo esa evaluación, y consecuentemente el poder realizarlo de una forma fiable, con una metodología sencilla y atractiva, basada en una metodología activa.

(cc)) BY-NC-ND 2017, UniversitatPolitècnica de València 
$\mathrm{Y}$ en un segundo nivel y considerando que actualmente se disponen de herramientas de evaluación a nivel institucional, contrastar los resultados con la herramienta desarrollada por los autores.

\section{Desarrollo de la innovación}

Considerando como principal objetivo, el definido en el apartado anterior, la evaluación de la competencia comunicación oral efectiva, se ha llevado a cabo durante los últimos años una metodología basada en la coevaluación para la obtención de resultados cuantitativos. La experiencia se ha llevado a cabo en la titulación del Grado en Ingeniería Química en la asignatura Ciencia de Materiales y más concretamente en las sesiones de laboratorio de la misma.

La metodología empleada para la evaluación y las tareas a desarrollar por el alumno en esta experiencia, están basadas en metodologías activas enfocadas hacia el aprendizaje del alumno. Esta experiencia tiene una doble intención, en primer lugar la evaluación de la competencia comunicación oral efectiva y en segundo lugar, e igual de importante, motivar a los alumnos a participar más activamente en el aprendizaje continuo en la asignatura.

En particular, en esta asignatura hay matriculados 90 alumnos este año, de ese modo y aunque las presentaciones orales son individuales, el trabajo de elaboración de la presentación se realiza en grupos formados por 4-5 estudiantes. Los estudiantes tienen que preparar una presentación con una duración comprendida entre 10-15 minutos. En dicha presentación los alumnos deberán exponer a sus compañeros un resumen de lo visto en la sesión anterior. La preparación de la tarea requiere realizar una síntesis de los aspectos y conclusiones más relevantes, así como el esfuerzo de estructurar y explicar dichos contenidos al resto de compañeros. De este modo, se busca que los alumnos lleven un seguimiento más continuo de la asignatura, así como se espera que consigan una comprensión más profunda que les ayude en el aprendizaje del contenido.

Las presentaciones orales fueron evaluadas por un lado por el profesor y por otro lado por los compañeros de clase, utilizando la misma herramienta de evaluación, la rúbrica desarrollada.

La evaluación se realizó utilizando dos rúbricas diferentes: la primera de ellas desarrollada por los autores como parte del "Proyecto PIME B12/14", ver Tabla 1, y que fue utilizada por alumnos y profesor. Y la segunda es la institucional (ver Tabla 2), utilizada por el profesor, que fue desarrollada posteriormente por un grupo de profesores expertos de la UPV, basándose en su propia experiencia y en los resultados obtenidos por otros profesores participantes en los proyectos PIME de la UPV. 
Tabla 1. Rúbrica comunicación oral desarrollada por los autores

\begin{tabular}{|c|c|c|c|c|c|c|c|}
\hline & CRITERIOS & 0-No cumple & 1-Deficiente & 2-Regular & 3-Bueno & 4-Muy bueno & 5-Excelente \\
\hline 1 & $\begin{array}{l}\text { Realiza una } \\
\text { introducción } \\
\text { efectiva al tema }\end{array}$ & no la realiza & $\begin{array}{l}\text { realiza } \\
\text { introducción } \\
\text { mayoritariamente } \\
\text { incompleta }\end{array}$ & $\begin{array}{l}\text { realiza } \\
\text { introducción } \\
\text { incompleta }\end{array}$ & $\begin{array}{l}\text { realiza } \\
\text { introducción }\end{array}$ & $\begin{array}{l}\text { realiza } \\
\text { introducción y } \\
\text { pone en situación } \\
\text { a la audiencia }\end{array}$ & $\begin{array}{l}\text { realiza } \\
\text { introducción } \\
\text { indicando } \\
\text { ejemplos del } \\
\text { interés del tema }\end{array}$ \\
\hline 2 & $\begin{array}{l}\text { Señala los objetivos } \\
\text { e ideas principales }\end{array}$ & no lo realiza & $\begin{array}{l}\text { señala alguno de } \\
\text { los objetivos }\end{array}$ & $\begin{array}{l}\text { señala los } \\
\text { objetivos de forma } \\
\text { incompleta }\end{array}$ & $\begin{array}{l}\text { señala todos los } \\
\text { objetivos }\end{array}$ & $\begin{array}{l}\text { señala todos los } \\
\text { objetivos de forma } \\
\text { organizada y } \\
\text { sintética }\end{array}$ & $\begin{array}{l}\text { señala todos los } \\
\text { objetivos de forma } \\
\text { organizada y } \\
\text { sintética, con un } \\
\text { enfoque aplicado }\end{array}$ \\
\hline 3 & $\begin{array}{l}\text { Presenta resultados } \\
\text { fundamentados } \\
\text { adecuadamente }\end{array}$ & no presenta & $\begin{array}{l}\text { presenta algún } \\
\text { resultado }\end{array}$ & $\begin{array}{l}\text { presenta de forma } \\
\text { incompleta }\end{array}$ & $\begin{array}{l}\text { presenta todos los } \\
\text { resultados }\end{array}$ & $\begin{array}{l}\text { presenta los } \\
\text { resultados } \\
\text { relevantes }\end{array}$ & $\begin{array}{l}\text { presenta los } \\
\text { resultados } \\
\text { relevantes y los } \\
\text { fundamenta }\end{array}$ \\
\hline 4 & $\begin{array}{l}\text { Conclusiones } \\
\text { apropiadas y } \\
\text { sintéticas }\end{array}$ & no las realiza & $\begin{array}{l}\text { señala alguna } \\
\text { conclusión }\end{array}$ & $\begin{array}{l}\text { señala } \\
\text { conclusiones de } \\
\text { forma incompleta }\end{array}$ & $\begin{array}{l}\text { señala todas las } \\
\text { conclusiones }\end{array}$ & $\begin{array}{l}\text { señala las } \\
\text { conclusiones más } \\
\text { relevantes } \\
\text { organizaday y } \\
\text { sintéticamente }\end{array}$ & $\begin{array}{l}\text { señala las } \\
\text { conclusiones más } \\
\text { relevantes de } \\
\text { forma organizada, } \\
\text { sintética y } \\
\text { evalúa/analiza sus } \\
\text { consecuencias }\end{array}$ \\
\hline 5 & $\begin{array}{l}\text { Interpreta, } \\
\text { argumenta y } \\
\text { justifica la } \\
\text { información } \\
\text { presentada }\end{array}$ & no lo realiza & $\begin{array}{l}\text { Lo realiza de } \\
\text { manera errónea }\end{array}$ & $\begin{array}{l}\text { Lo realiza bien } \\
\text { pero de manera } \\
\text { muy limitada }\end{array}$ & $\begin{array}{l}\text { Lo realiza bien } \\
\text { pero incompleto }\end{array}$ & $\begin{array}{l}\text { Lo realiza de } \\
\text { manera correcta }\end{array}$ & $\begin{array}{l}\text { Lo realiza } \\
\text { correctamente, y } \\
\text { enfatizando los } \\
\text { aspectos } \\
\text { relevantes }\end{array}$ \\
\hline 6 & \begin{tabular}{|l} 
Presentación \\
estructurada, clara, \\
coherente y eficaz
\end{tabular} & no cumple ninguna & $\begin{array}{l}\text { parcialmente } \\
\text { estructurada }\end{array}$ & es estructurada & $\begin{array}{l}\text { al menos es } \\
\text { estructurada y } \\
\text { clara }\end{array}$ & $\begin{array}{l}\text { al menos es } \\
\text { estructurada, clara } \\
\text { y coherente }\end{array}$ & $\begin{array}{l}\text { al menos es } \\
\text { estructurada, } \\
\text { clara, coherente y } \\
\text { eficaz }\end{array}$ \\
\hline 7 & $\begin{array}{l}\text { Utiliza un lenguaje } \\
\text { técnico adecuado }\end{array}$ & nunca & $\begin{array}{l}\text { lo usa con errores } \\
\text { reiterados }\end{array}$ & $\begin{array}{l}\text { lo usa con algún } \\
\text { error }\end{array}$ & $\begin{array}{l}\text { lo utiliza } \\
\text { ocasionalmente }\end{array}$ & $\begin{array}{l}\text { lo utiliza habitual y } \\
\text { adecuadamente }\end{array}$ & $\begin{array}{l}\text { lo utiliza habitual y } \\
\text { adecuadamente, e } \\
\text { introduce nuevos } \\
\text { conceptos }\end{array}$ \\
\hline 8 & $\begin{array}{l}\text { Utiliza los recursos } \\
\text { disponibles para una } \\
\text { comunicación más } \\
\text { eficiente }\end{array}$ & $\begin{array}{l}\text { no utiliza los } \\
\text { recursos } \\
\text { disponibles }\end{array}$ & $\begin{array}{l}\text { utiliza los recursos } \\
\text { de forma } \\
\text { inadecuada }\end{array}$ & $\begin{array}{l}\text { utiliza los recursos } \\
\text { pero no para } \\
\text { clarificar ideas }\end{array}$ & $\begin{array}{l}\text { utiliza los recursos } \\
\text { para clarificar las } \\
\text { ideas principales }\end{array}$ & $\begin{array}{l}\text { utiliza los recursos } \\
\text { para clarificar las } \\
\text { ideas de forma } \\
\text { generalizada }\end{array}$ & $\begin{array}{l}\text { utiliza los recursos } \\
\text { disponiblese e } \\
\text { introduce nuevos } \\
\text { para clarificar las } \\
\text { ideas de forma } \\
\text { generalizada }\end{array}$ \\
\hline 9 & $\begin{array}{l}\text { La presentación se } \\
\text { ajusta al tiempo } \\
\text { disponible }\end{array}$ & $\begin{array}{l}\text { Ningún control } \\
\text { temporal de la } \\
\text { extensión parcial y } \\
\text { total }\end{array}$ & $\begin{array}{l}\text { Se excede o le } \\
\text { sobra demasiado } \\
\text { tiempo }\end{array}$ & $\begin{array}{l}\text { Se ajusta al tiempo } \\
\text { disponible de } \\
\text { forma aproximada }\end{array}$ & $\begin{array}{l}\text { Se ajusta al tiempo } \\
\text { disponible }\end{array}$ & $\begin{array}{l}\text { Se ajusta al tiempo } \\
\text { disponible, dedica } \\
\text { el tiempo } \\
\text { apropiado en cada } \\
\text { parte }\end{array}$ & $\begin{array}{l}\text { Se ajusta al tiempo } \\
\text { disponible, dedica } \\
\text { el tiempo } \\
\text { apropiado en cada } \\
\text { parte y se } \\
\text { reorganiza en caso } \\
\text { necesario }\end{array}$ \\
\hline 10 & $\begin{array}{l}\text { Dicción clara, sin } \\
\text { muletillas, tono } \\
\text { adecuado, postura } \\
\text { corporal adecuada y } \\
\text { contacto visual }\end{array}$ & no cumple ninguna & cumple 1 & $\begin{array}{l}\text { Dicción clara y } \\
\text { tono monótono }\end{array}$ & $\begin{array}{l}\text { Dicción clara y } \\
\text { postura corporal }\end{array}$ & $\begin{array}{l}\text { Dicción clara, } \\
\text { postura corporal y } \\
\text { tono adecuado }\end{array}$ & $\begin{array}{l}\text { Dicción clara, } \\
\text { postura corporal, } \\
\text { tono adecuado y } \\
\text { contacto visual }\end{array}$ \\
\hline 11 & $\begin{array}{l}\text { Analiza, valora y } \\
\text { responde a las } \\
\text { preguntas que se } \\
\text { formulan }\end{array}$ & \begin{tabular}{|l} 
No cumple \\
ninguna
\end{tabular} & $\begin{array}{l}\text { Analiza, pero ni } \\
\text { valora ni responde }\end{array}$ & $\begin{array}{l}\text { Analiza y valora, } \\
\text { pero no responde }\end{array}$ & $\begin{array}{l}\text { Analiza, valora y } \\
\text { responde de } \\
\text { manera limitada, } \\
\text { con ayuda del } \\
\text { profesor }\end{array}$ & $\begin{array}{l}\text { Analiza, valora y } \\
\text { responde con } \\
\text { enfoque propio }\end{array}$ & $\begin{array}{l}\text { Analiza, valora y } \\
\text { responde con } \\
\text { enfoque propio, y } \\
\text { plantea cuestiones } \\
\text { relacionadas }\end{array}$ \\
\hline
\end{tabular}


Tabla 2. Rúbrica comunicación oral UPV

\begin{tabular}{|c|c|c|c|c|}
\hline \multirow{2}{*}{ INDICADORES } & \multicolumn{4}{|c|}{ DESCRIPTORES } \\
\hline & D. No alcanzado & C. En desarrollo & B. Bien /adecuado & A. Excelente/ejemplar \\
\hline $\begin{array}{l}\text { Muestra una } \\
\text { disposición personal } \\
\text { positiva hacia la } \\
\text { comunicación }\end{array}$ & $\begin{array}{l}\text { No se implica en las } \\
\text { actividades propuestas o } \\
\text { lo hace faltondo a las } \\
\text { normas básicas de } \\
\text { educación y convivencia }\end{array}$ & $\begin{array}{l}\text { Se implica mínimamente } \\
\text { y/o lo hace manifestando } \\
\text { actitudes poco apropiados }\end{array}$ & $\begin{array}{l}\text { Muestra una disposición } \\
\text { personal positiva hacia } \\
\text { la comunicación }\end{array}$ & $\begin{array}{l}\text { Sus presentaciones } \\
\text { voluntarias fomentan la } \\
\text { participación de oftos y } \\
\text { aportan valor añadido }\end{array}$ \\
\hline $\begin{array}{l}\text { Realiza exposiciones } \\
\text { interesantes y } \\
\text { convincentes, que } \\
\text { desarrolla cuando le } \\
\text { formulan preguntas }\end{array}$ & $\begin{array}{l}\text { Es posible que exponga } \\
\text { algunas ideas } \\
\text { importantes, pero no logra } \\
\text { captar la atención y, ante } \\
\text { las preguntas, a menudo, } \\
\text { no responde o lo hace de } \\
\text { manera incorrecta }\end{array}$ & $\begin{array}{l}\text { Expone solo algunas ideas } \\
\text { importantes y logra captar } \\
\text { la atención } \\
\text { intermitentemente. Ante las } \\
\text { preguntas, sus respuestos } \\
\text { repiten la presentación, } \\
\text { pero no la amplían }\end{array}$ & $\begin{array}{c}\text { Realiza exposiciones } \\
\text { interesantes y } \\
\text { convincentes, que } \\
\text { apoya y desarrolla } \\
\text { cuando le formulan } \\
\text { preguntas }\end{array}$ & $\begin{array}{l}\text { Su exposición de ideas } \\
\text { resulta completa, } \\
\text { interesante y convincente. } \\
\text { Sus respuestas generan } \\
\text { nuevas intervenciones } \\
\text { (preguntas) }\end{array}$ \\
\hline $\begin{array}{l}\text { La exposición está } \\
\text { debidamente } \\
\text { preparada y se ajusta } \\
\text { al tiempo establecido }\end{array}$ & $\begin{array}{l}\text { La exposición no está } \\
\text { debidamente } \\
\text { estructurada y/o no se } \\
\text { ajusta al tiempo } \\
\text { establecido }\end{array}$ & $\begin{array}{l}\text { La exposición está } \\
\text { organizada, pero hay } \\
\text { demasiada o escasa } \\
\text { información y/o no se ajusto } \\
\text { al tiempo establecido }\end{array}$ & $\begin{array}{l}\text { La audiencia puede } \\
\text { captar claramente la } \\
\text { eficaz estructura del } \\
\text { contenido y la } \\
\text { exposición se ajusto al } \\
\text { tiempo establecido }\end{array}$ & $\begin{array}{c}\text { La selección y organización } \\
\text { del contenido y su } \\
\text { ilustración (ejemplos, } \\
\text { analogías...) son coherentes } \\
\text { y la exposición se ajusta al } \\
\text { tiempo establecido }\end{array}$ \\
\hline $\begin{array}{l}\text { Respeta las normas } \\
\text { lingǘsticas } \\
\text { (corrección) y se } \\
\text { expresa de manera } \\
\text { correcta (claridad) }\end{array}$ & $\begin{array}{l}\text { No realiza un buen uso del } \\
\text { lenguaje y/o no utiliza la } \\
\text { terminología específica } \\
\text { de la materia }\end{array}$ & $\begin{array}{l}\text { La presentación no es fluida } \\
\text { (lenguaje limitado] y utiliza } \\
\text { escasa terminología } \\
\text { especifica o lo hace } \\
\text { inapropiadamente }\end{array}$ & $\begin{array}{c}\text { Respeta las normas } \\
\text { lingúisticas y se expresa } \\
\text { de manera correcto, } \\
\text { utilizando de modo } \\
\text { pertinente la } \\
\text { terminología especifica } \\
\text { de la materia }\end{array}$ & $\begin{array}{l}\text { La exposición se adapta a } \\
\text { la audiencia, haciendo uso } \\
\text { de un lenguaje y de una } \\
\text { terminología específicos, } \\
\text { apropiados, precisos y ricos }\end{array}$ \\
\hline $\begin{array}{l}\text { La comunicación no } \\
\text { verbal es apropiada }\end{array}$ & $\begin{array}{l}\text { Su lenguaje no verbal no } \\
\text { respalda y/o distrae su } \\
\text { discurso verbal }\end{array}$ & $\begin{array}{l}\text { Su lenguaje no verbal } \\
\text { muestra alguna } / \mathrm{s} \\
\text { deficiencia } / \mathrm{s}\end{array}$ & $\begin{array}{l}\text { Su lenguoje no verbal es } \\
\text { coherente con el verbal }\end{array}$ & $\begin{array}{l}\text { Su lenguaje no verbal } \\
\text { refuerza el verbal: gestos } \\
\text { firmes, ritmo variado y } \\
\text { entonación intencional }\end{array}$ \\
\hline $\begin{array}{l}\text { Refuerza sus ideas } \\
\text { con la ayuda de los } \\
\text { medios de apoyo }\end{array}$ & $\begin{array}{l}\text { No utiliza los medios de } \\
\text { apoyo apropiados o hace } \\
\text { un mal uso de los mismos }\end{array}$ & $\begin{array}{l}\text { No siempre utiliza los medios } \\
\text { de apoyo más apropiados } \\
\text { o no hace el mejor uso de } \\
\text { los mismos }\end{array}$ & $\begin{array}{l}\text { Los medios de apoyo } \\
\text { contribuyen a que el } \\
\text { discurso se comprenda } \\
\text { mejor, enfatizando sus } \\
\text { puntos clave }\end{array}$ & $\begin{array}{c}\text { Los medios de apoyo } \\
\text { destacan por su } \\
\text { calidad/originalidad y } \\
\text { contribuyen a que el } \\
\text { disourso se comprenda } \\
\text { mejor }\end{array}$ \\
\hline
\end{tabular}

Posteriormente la idea es realizar una comparación entre ambas herramientas, viendo si hay una buena correlación entre ambas, pudiendo confirmar en ese caso que hay una buena fiabilidad en los resultados obtenidos previamente en el proyecto PIME llevado a cabo. La rúbrica PIME, es la que se utilizó inicialmente como parte de este estudio y fue diseñada y desarrollada por los autores [Carballeira (2016)]. De otro lado, la rúbrica UPV recoge los siguientes items para la evaluación de la competencia: actitud en la comunicación; selección de contenido y organización; estructura y ajuste al tiempo disponible; uso del lenguaje científico; comunicación no verbal y uso de medios de apoyo. Cada uno de estos items se gradúa en cuatro grados o niveles de consecución: A. Excelente, B. Adecuado, C. En desarrollo y D. No alcanzado.

Para poder hacer la comparación entre ambas herramientas de evaluación, se realizó una correspondencia entre los items evaluados en cada una de las rúbricas, debido a que se encontró un ítem o un grupo de items en la rúbrica de los autores (rúbrica PIME) que también se encuentra como ítem evaluable en la rúbrica UPV. A continuación se detallan los ítems evaluados en la rúbrica UPV y entre paréntesis los correspondientes en la rúbrica de la Tabla 1: exposición interesante y respuesta a preguntas (ítems 5 y 11); estructura y ajuste al tiempo disponible (ítems 6 y 9); uso del lenguaje científico (ítem 7); comunicación no verbal (ítem 10) y uso de medios de apoyo (ítems 8). 
Para poder hacer la comparación entre ambos sistemas de evaluación, fue necesario hacer una correspondencia numérica y la normalización de los valores para cada aspecto evaluado. A los ítems de la rúbrica UPV se les asignó un puntuación del 1 al 4, siendo 1 el nivel no alcanzado y el 4 el nivel excelente. Posteriormente esas puntuaciones se normalizaron a una escala de 5 puntos si hay correspondencia con un ítem de la rúbrica PIME o de 10 puntos en el caso de haber dos ítems. Finalmente, mediante la suma de la puntuación de todos los ítems se obtuvo la valoración de cada uno de los actos de evaluación con las dos rúbricas y se procedió a su comparación.

\section{Resultados}

De acuerdo con el trabajo previo realizado por los autores en la evaluación de las competencias transversales [Sahuquillo (2015), Sonseca (2015)], a continuación se resumen los resultados más relevantes. En la evaluación realizada por los alumnos utilizando la rúbrica mostrada en la Tabla 1, se observaron pequeñas diferencias en las evaluaciones realizadas entre los diferentes alumnos para un mismo acto de evaluación. De este modo se puede deducir que los usuarios de la herramienta no tuvieron ningún problema en su utilización ni en la comprensión de los criterios a valorar, de este modo se puede considerar que la rúbrica desarrollada en el proyecto PIME es una herramienta de evaluación sencilla pues se consigue una valoración uniforme entre usuarios no expertos como pueden ser los alumnos.

En el estadio inicial de la evaluación de la competencia transversal y para comprobar la bondad de la rúbrica, se llevaron a cabo valoraciones a cargo de dos profesores para cada uno de los actos de evaluación. De los resultados se obtuvieron diferencias mínimas entre las evaluaciones realizadas por los dos profesores, con diferencias incluso inferiores a las encontradas entre las valoraciones de los alumnos [Sonseca (2015)].

En la Fig. 1 (a) y (b), se muestran a modo de ejemplo la comparativa entre las calificaciones de alumnos y profesores para un mismo acto de evaluación, mostrándose los resultados obtenidos en dos años académicos diferentes.

A la vista de estos resultados se puede deducir que hay una buena correlación entre las evaluaciones realizadas por los alumnos y por los profesores, siendo como es obvio las calificaciones de los alumno superiores a las de los profesores, pero a pesar de ese detalle, sin mucha diferencia entre la valoración entre los distintos evaluadores. Indicando por tanto que los criterios de evaluación son fácilmente comprensibles y claros para los distintos evaluadores, profesores y alumnos, incluso aunque no se trate de evaluadores expertos. Este hecho permite considerar a priori la rúbrica desarrollada como una herramienta de evaluación válida, permitiendo por tanto y en base a los resultados obtenidos involucrar a los alumnos como parte de la evaluación de la asignatura.

(c)) EY-NC-ND 2017, UniversitatPolitècnica de València 


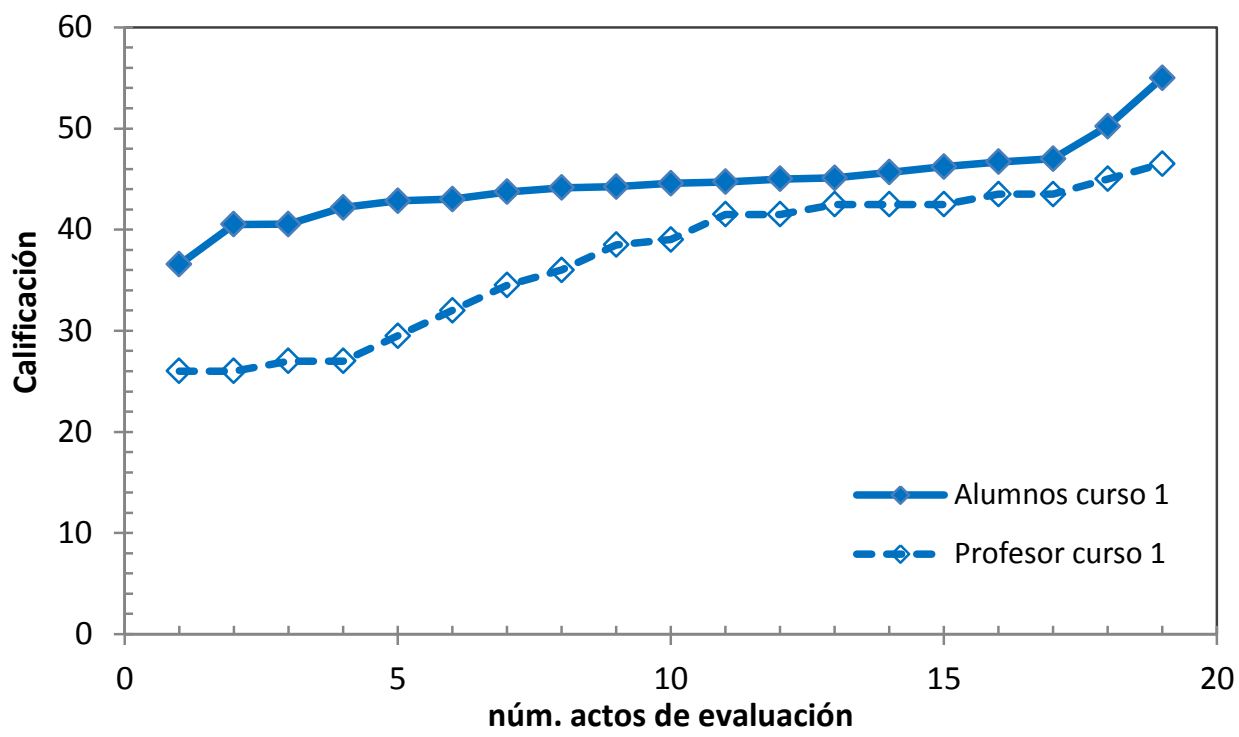

(a)

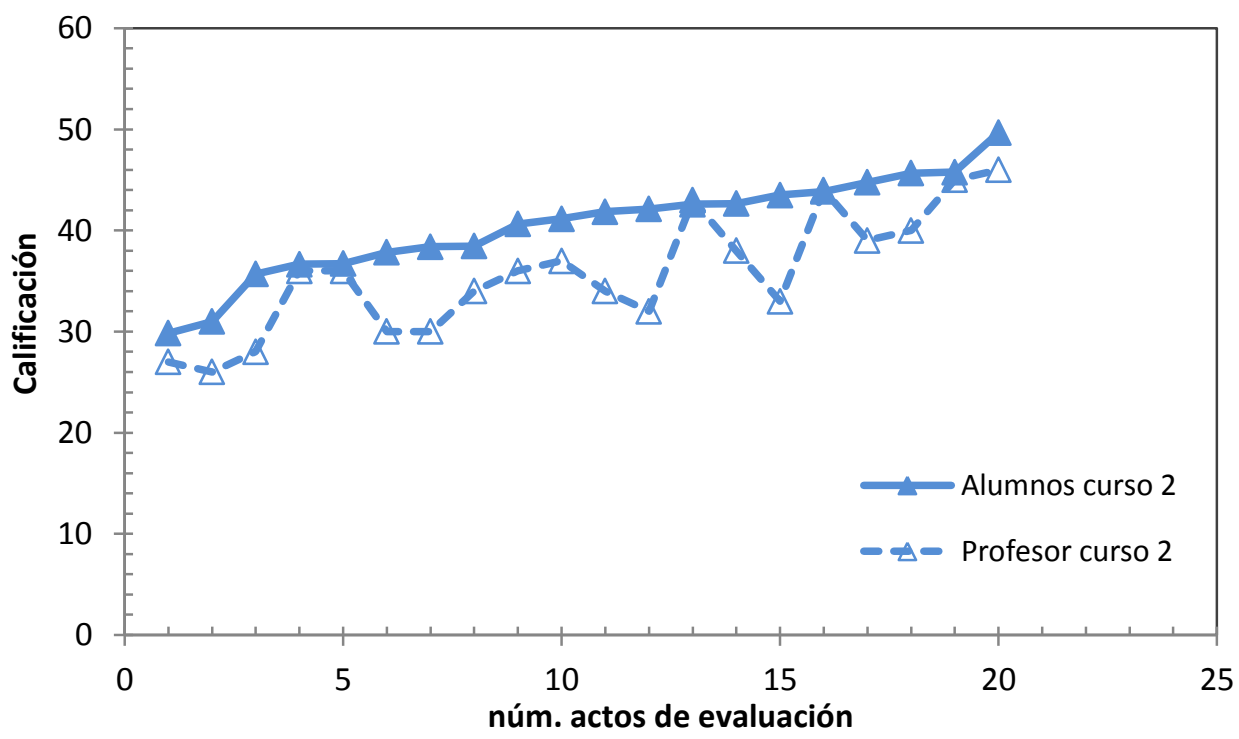

(b)

Fig. 1 Comparativa calificaciones alumnos y profesor (a) curso académico 1 y (b) curso académico 2.

A partir de los resultados obtenidos en el proyecto PIME, parece que se cuenta con un herramienta de evaluación sólida, pero con la intención de intentar confirmarlo se busca el comparar los resultados de la rúbrica que se desarrollo durante el PIME con la rúbrica que se desarrolló posteriormente en la UPV. Para ello se ha procedido a evaluar por parte del profesor un mismo acto de evaluación con las rúbricas mostradas en la Tabla 1 y 2 . Se evaluaron un total de 19 actos de evaluación, para la presentación de los resultados se ha 
procedido a ordenar las calificaciones de menor a mayor para una presentación y visualización de los resultados más sencilla, ver Fig. 2. En el gráfico se comparan los resultados obtenidos por el profesor con las dos rúbricas, teniendo en cuenta que la calificación máxima posible es de 35 puntos.

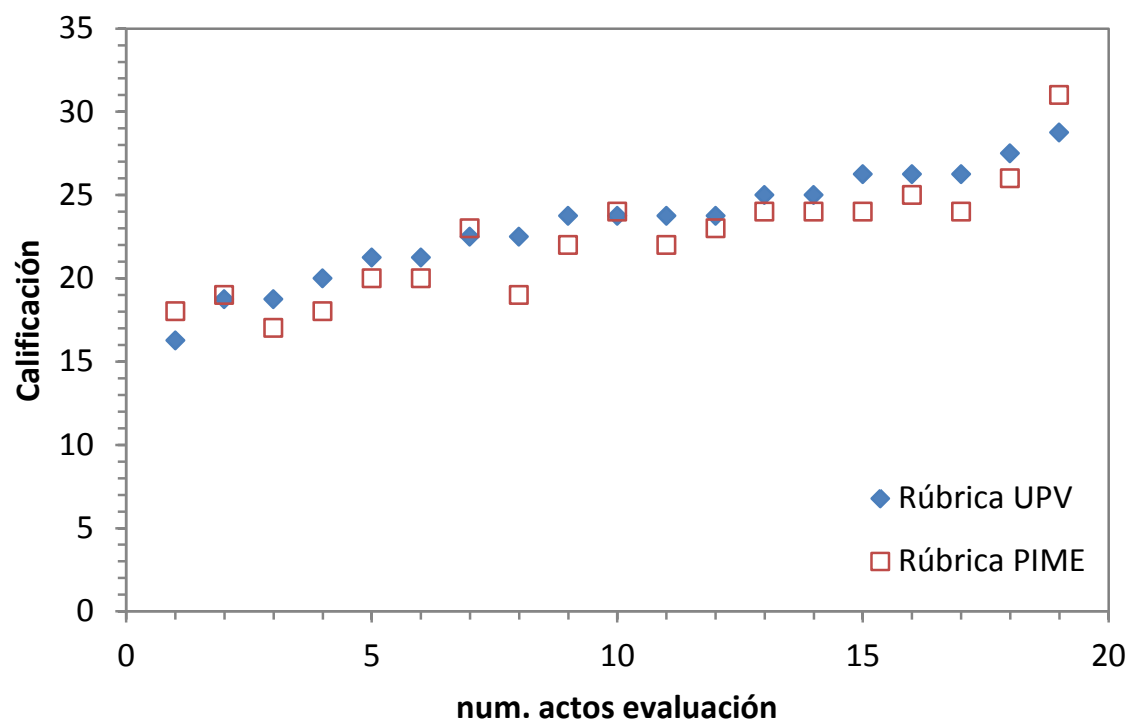

Fig. 2 Comparativa calificaciones profesor con dos rúbricas diferentes

En todos los actos de evaluación realizados, se obtuvieron pequeñas diferencias entre las valoraciones realizadas por el profesor empleando las dos rúbricas. De las 19 valoraciones, 14 de ellas, cerca del 75\% de los casos, presentan diferencias entre las valoraciones de las dos rúbricas inferiores o iguales al 5\% (en porcentaje y relativo a los 35 puntos totales). También se observa que en general, las calificaciones son superiores en la rúbrica UPV, quizá debido a que hay definidos cuatro niveles de consecución en lugar de los seis de la rúbrica PIME.

De este modo y dado que las diferencias entre las rúbrica utilizadas son muy pequeñas, y teniendo en cuenta que la rúbrica UPV fue desarrollada por un conjunto de profesores multidisciplinar y con experiencia en este campo, se puede concluir que la rúbrica desarrollada en el PIME se confirma como una herramienta que permite obtener resultados consistentes y fiables para la evaluación de la competencia comunicación oral efectiva. Además las pequeñas diferencias obtenidas en las valoraciones entre los diferentes posibles evaluadores, alumno-alumno, profesor-profesor y alumno-profesor, en el proceso de coevaluación con la rúbrica UPV, dan validez, consistencia a la metodología y a la herramienta de evaluación desarrollada, permitiendo incorporar al alumno en el proceso de evaluación e intentar que tomen conciencia de su nivel de consecución y qué aspectos revisar con el objetivo de mejorar sus competencias.

(c) BY-NC-ND 2017, UniversitatPolitècnica de València 


\section{Conclusiones}

En la etapa previa del estudio, con la rúbrica PIME, se obtuvieron mínimas diferencias entre las evaluaciones realizadas por dos profesores. Con diferencias algo superiores entre las valoraciones entre los alumnos y una buena concordancia entre las evaluaciones realizadas por profesores y alumnos. Todo ello lleva a pensar que la herramienta desarrollada para la evaluación era sencilla y reporta resultados fiables aún siendo utilizada por evaluadores no experimentados como los alumnos.

La buena correlación entre las evaluaciones permite deducir que los criterios a evaluar son fácilmente comprensibles y claros para los distintos evaluadores, profesores y/o alumnos. Esto permite considerarla como una herramienta de evaluación válida, permitiendo involucrar a los alumnos como parte de la evaluación de la asignatura.

La comparación de la evaluación realizada con la rúbrica desarrollada en el PIME y la institucional reporta resultados muy similares y por tanto reproducibles independientemente de la rúbrica utilizada.

Los resultados obtenidos con la rúbrica desarrollada en el PIME, en base a las pequeñas diferencias entre las valoraciones realizadas por distintos evaluadores, así como con la rúbrica institucional, permite concluir que se trata de una herramienta valida, fiable y consistente para la evaluación de la competencia.

Respecto a la metodología, el hecho de hacer exponer a los estudiantes al trabajo realizado, y convertirlos en evaluadores, les hace ser más conscientes de su nivel de logro y de qué pueden hacer para mejorar sus competencias.

\section{Agradecimientos}

El proyecto has sido financiado por el Vicerrectorado de Estudios, Calidad y Acreditación de la Universitat Politècnica de València a través del programa PIME 2016-17 bajo la referencia A26.

\section{Referencias}

CARBALlEIRA, J., MARTÍNEZ, J., SAHUQUILlO, O., SONSECA, A., DENIA, F.D., SUÑER, J.L., VILA, P., RÓDENAS, J.J., MARCO, M. (2015). "Desarrollo de estrategias de evaluación de competencias transversales en asignaturas de ingeniería mecánica y de materiales" en Congreso Nacional de Innovación Educativa y Docencia en Red (IN-RED 2015). Valencia: Universitat Politècnica de València. (923 - 937).

CARBALLEIRA, J., MARTÍNEZ-CASAS, J., SAHUQUILLO, O., SONSECA, A., SUÑER, J.L., VILA, P., DENIA, F. D., RÓDENAS, J.J., MARCO, O. (2016). "Assessment of problem-solving skills and capacity for applying knowledge in practice in subjects related to mechanical and materials engineering" en Advances in Higher Education, Ed. Universitat Politècnia de València, Chapter 5, pp. 71-88. 
RIECKMANN, M. (2012). Future-oriented higher education: Which key competencies should be fostered through university teaching and learning? en Futures, 44, 127-135.

SURSOCK, H. S. (2010). A decade of change in european higher education. Trends 2010. Brussels: European University Association.

SAHUQUILLO, O., SONSECA, A., MARTÍNEZ, J., CARBALLEIRA, J., DENIA, F.D., RÓDENAS, J.J., MARCO, M. (2015). Propuesta de evaluación de las competencias de comunicación oral y escrita en asignaturas de Ingeniería Mecánica y Ciencia de Materiales en Congreso Nacional de Innovación Educativa y Docencia en (IN-RED 2015). Valencia: Universitat Politècnica de València. (973 - 987)

SONSECA, A., SAHUQUILlO, O., MARTÍNEZ, J., CARBALLEIRA, J., DENIA, F.D., RÓDENAS J.J. (2015). Assessment of oral and written communication competences in the European Higher Education Area: a proposal of evaluation methodologies in 1st International Conference on Higher Education Advances (HEAd'15). Valencia, Spain: Editorial Universitat Politècnica de València. (2 - 9).

TUNING PROJECT. (2014). Approaches to teaching, learning and assessment in competences based degree programmes. http://www.unideusto.org/tuningeu/teachinglearning-a-assessment.html [Consulta: 23 Marzo 2017]

UPV (2014). Competencias Transversales. http://competencias.webs.upv.es/wp/ [Consulta: 21 Marzo 2017] 